\title{
Trastorno de la comunicación social (pragmático): ¿síndrome o síntoma?
}

\section{Social (pragmatic) communication disorder: syndrome or symptom?}

\author{
Mónica González Blanco, Rosa M. Rivas Torres y Santiago López Gómez
}

Dpto. Psicología Evolutiva y de la Educación, Universidad de Santiago de Compostela

\begin{abstract}
Resumen:
Las dificultades pragmáticas repercuten en la comunicación social funcional eficaz del sujeto que las padece, en su participación social, en sus relaciones y logros. El Trastorno de la comunicación social (pragmático) (DSM-5) es un trastorno poco conocido y que describe una gran imprecisión. Se hace un estudio bibliográfico de las publicaciones que versan sobre el TCS (años 2000-2014) en el WOS. Los resultados obtenidos, muestran que las dificultades pragmáticas repercuten en la comunicación social. La problemática con la pragmática es, en un gran número de estudios, encuadrada como sintomatología de otros trastornos, principalmente dentro de TEA e TEL. Este hecho se define por una fuerte comorbilidad entre el TCS y otros trastornos, tales como el TEL y el TEA que es necesario concretar a través de diagnósticos fiables y objetivos de las futuras investigaciones.
\end{abstract}

Palabras clave: Trastorno de la comunicación social. Trastorno pragmático. Sintomatología. Caracterización clínica.

\section{Introduccción}

Las habilidades pragmáticas se pueden observar a partir del uso que hace el hablante del lenguaje, para comunicarse en diferentes contextos y con distintos interlocutores (BaixauliFortela, Roselló y Miranda-Casas, 2004). Esto implica conocer y saber utilizar las reglas lingüísticas, pero conjuntamente tener conocimiento sobre el interlocutor, sobre el contexto y sobre los usos del lenguaje (Taboada, Rivas y López, 2012).

La pragmática se pone de manifiesto en las interacciones sociales. Tiene que ver con dos grandes cuestiones; por un lado con la capacidad para utilizar y compartir un código lingüístico entre hablante y oyente y, a su vez, disponer de la capacidad para poder comunicar, sea una idea, sea un hecho o un sentimiento. Tal y como pone de manifiesto Monfort (2009), en el desarrollo normal, la relación entre comunicación y lenguaje es bidireccional e interdependiente.

El trastorno de la comunicación social (TCS), o pragmático, es un trastorno poco conocido, si bien el número de sujetos que lo presentan es importante (Monfort, Juárez y Monfort, 2004).

El TCS se caracteriza por una dificultad primaria con la pragmática, o los usos sociales del lenguaje y la comunicación, que se manifiestan por déficits tanto en la comprensión como en el seguimiento de las normas sociales de la comunicación verbal y no verbal en contextos cotidianos, y en la falta de adaptación del lenguaje a las necesidades del oyente o de la situación y el seguimiento de las reglas de la comunicación y el discurso. Los déficits en la comunicación social se manifiestan a modo de limitaciones funcionales en la eficacia comunicativa, la participación social, el desarrollo de relaciones sociales, logros académicos o resultados laborales.
Los déficits no se explican mejor por pobres habilidades en los dominios de la estructura del lenguaje o las habilidades cognitivas.

El trastorno pragmático resulta ser menos preciso que los demás trastornos de la comunicación debido, sobre todo, a que sus manifestaciones no se observan dentro de cuestiones tan objetivables y medibles como la fonología o la sintaxis, sino que se hacen evidentes dentro de la heterogeneidad y amplitud que se expresa en la interacción social y dentro de toda la subjetividad que conlleva. Por tanto, implica, sobre todo, a las habilidades sociales y a la adaptación de la conducta en general. A esta imprecisión cabe añadir que considerando la presencia de otras posibles patologías en el niño, como TDAH, discapacidad intelectual, X frágil, TEA, etc., el perfil de dificultades pragmáticas podría variar. Este hecho, tal y como señalan Baixauli-Fortela et al. (2004), podría implicar la existencia de diferentes subtipos de dificultad pragmática. De tal manera se describe una fuerte comorbilidad entre el trastorno de la comunicación social o pragmático con otros trastornos, como pueden ser el TDAH (Tannock y Martinussen, 2001), o el X frágil (Belser y Dudhalter, 2001; Sudhalter, Morianon y Brooks, 1992), pero sobre todo con el TEA (Botting y Conti-Ramsden, 2003). Ahora bien, eso no impide que no se pueda observar el trastorno pragmático en ausencia de otros trastornos (Botting y Conti-Ramsden, 1999; Roqueta y Esteban, 2010).

\section{El TCS como categoría ¿síndrome o síntoma?}

El trastorno de la comunicación social adquiere categoría de trastorno, como tal, en el DSM-5 (APA, 2013), donde se definen los criterios diagnósticos en base a las dificultades registradas y, de manera general, se hace mención de las consecuencias que traen consigo las mismas.

Son escasas las investigaciones centradas en el análisis y estudio de la pragmática y su problemática. Las líneas de investigación se orientan mayoritariamente al trastorno específico del lenguaje (TEL) o la ligazón de éste con los trastornos encuadrados dentro del denominado espectro autista. Evidencia de ello la aporta el análisis pormenorizado de los artículos más citados en la Web of Science (WOS, de Thomson Reuters).

El proceso de búsqueda realizado en la WOS comenzó agregando cómo campo los términos en inglés "disorder pragmatic of social communication", tal como el TCS es denominado en el DSM-5 en su versión original. Las entradas resultantes se filtraron por fecha de publicación acotando la misma al período comprendido entre los años 2000 y 2014 (ambos incluidos). Las entradas anteriores al año 2000 son escasas (n: 32) y con apenas peso en el estudio. El número de estudios citados se reduce a 234. Posteriormente se procedió a la elaboración de un informe de citas que da cuenta del hecho de que, en el período seleccionado, los elementos publicados fluctuaron significativamente de un año al otro (Figura I). 

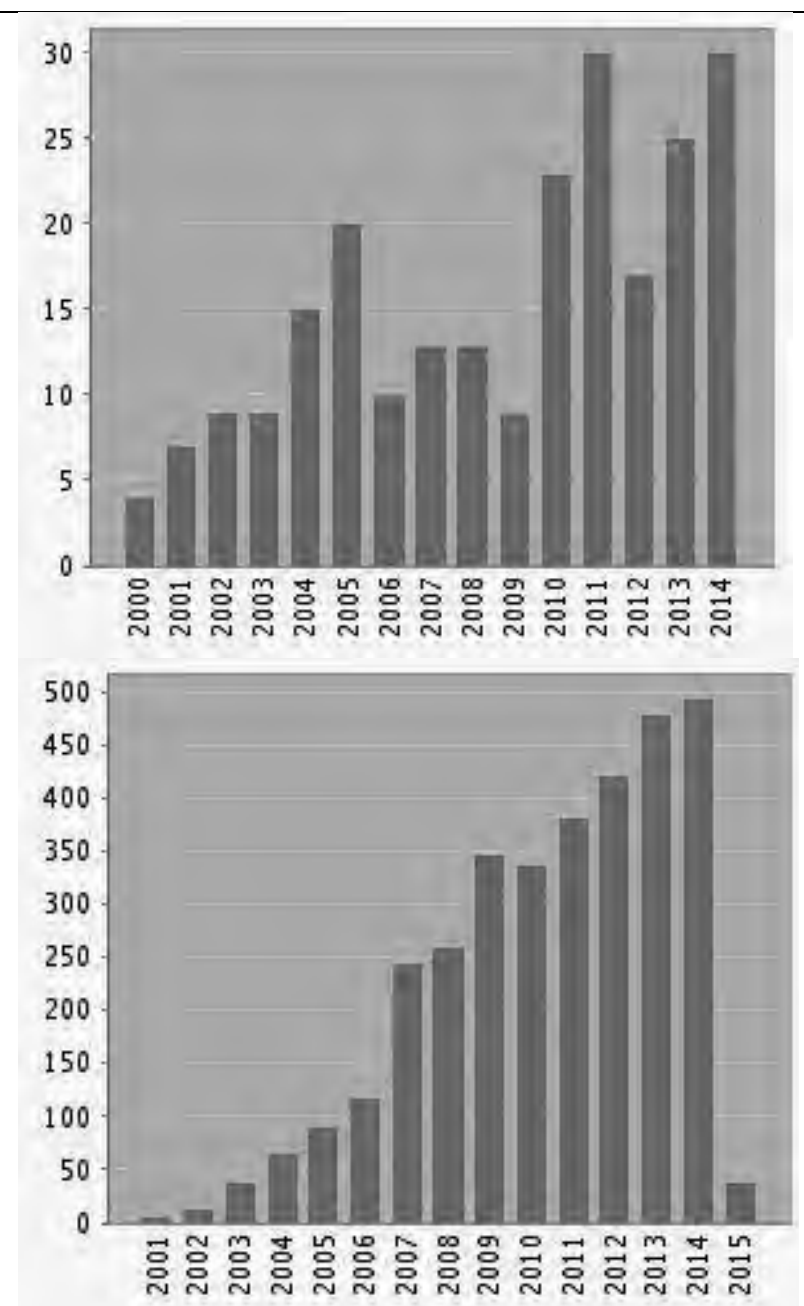

Figura I. Elementos publicados en el WOS sobre "disorder pragmatic of social communication” (2000-2014) y por número de citas.

Se observa un ascenso continuo en el número de informes presentados entre 2000-2005, excepto en el año 2003; posteriormente, descenso significativo en el 2006, ligera remontada en los dos años siguientes y nuevo descenso en el 2009 para sufrir una fuerte subida en el 2010 cuya tendencia alcista continua en el año posterior, siendo el 2011 el período en el que se presentaron más estudios relacionados con el TCS. Al año siguiente se reduce de manera importante el número de elementos, registrándose después una subida en el 2013 que continúa en el 2014. En cuanto al número de citas hechas cada año, estas en general van en aumento a medida que transcurre el tiempo, esto es, se registra un incremento continuo de los elementos citados desde el año 2000 hasta 2013, excepto en el 2010, lo que refleja el creciente interés por el tema.

Los diez artículos más citados vinculan el análisis de los déficits pragmáticos a alguna patología, ya sea al trastorno del espectro del autismo, trastorno específico del lenguaje, esquizofrenia, trastorno bipolar, síndrome de Williams, síndrome de Asperger, TDAH o lesión traumática. Ninguno de ellos se centra específicamente en el trastorno de comunicación social.

Son muchos los trabajos científicos que vinculan pragmática y autismo.Sometiendo el análisis a temática de cada uno de los 234 artículos científicos, mediante la consulta de los resúmenes, se procedió primero al descarte de 14 de los trabajos por diferentes motivos: no estaban ni en lengua inglesa ni castellana o la temática no mantenía relación con el objeto de estudio que nos ocupa. No se procedió a considerar cómo criterio de exclusión la edad de los participantes en estas investigaciones, bien porque en la consulta de los resúmenes no en todos los casos figuraba este dato bien porque se considera relevante el estudio del influjo y de las repercusiones que las dificultades y carencias pragmáticas tiene en patologías como la esquizofrenia y Parkinson, las cuáles se manifiestan en la adultez y vejez.

De los 220 artículos finalmente analizados se detectó que un amplio porcentaje, en concreto, un 35\% (n: 77) de los mismos relacionaban las alteraciones pragmáticas con los trastornos de espectro autista. Asimismo, un 14\% (n: 30) de los estudios giraban alrededor de los problemas pragmáticos bien definiendo el concepto de pragmática, bien detallando las repercusiones que traen consigo las alteraciones en este campo, bien su estrecha vinculación con otros trastornos del lenguaje. Por otra parte, son también significativos, pues representan un $9 \%$, (n: 19) el número de investigaciones que vinculan los déficits pragmáticos con lesiones cerebrales adquiridas o con el trastorno específico del lenguaje respectivamente.

Las investigaciones más recientes se centran, en buena parte de los casos, en el estudio de los síntomas pragmáticos en menores con un diagnóstico de TEA. Por tanto, la consideración que se le da a las alteraciones en la pragmática es la de síntoma del propio TEA. Este es incluso el etiquetado que se le concede en aquellos trabajos que analizan los déficits pragmáticos en sujetos con TEL, esquizofrenia o síndrome de Asperger, entre otros. En el caso del estudio de pacientes que sufrieron lesiones cerebrales el foco de atención recae habitualmente en las correlaciones que dicha lesión tienen en el comportamiento pragmático

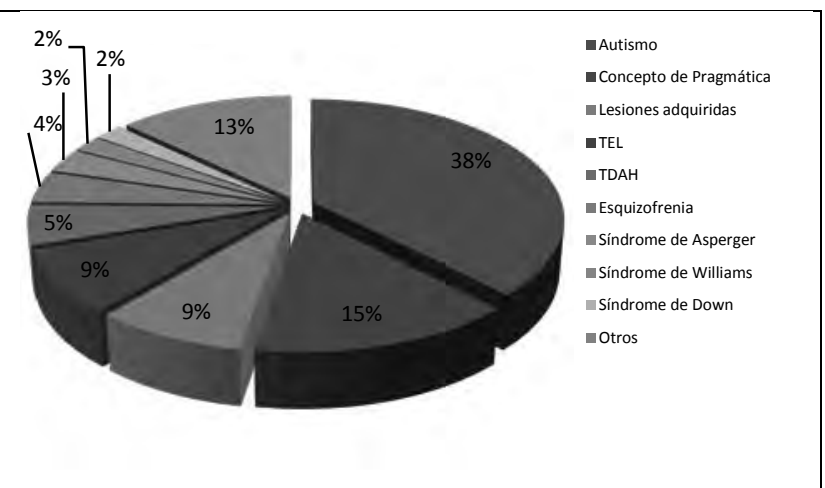

Figura II. Ejes temáticos presentes en los artículos científicos analizados

Por otra parte, la necesidad de elaborar herramientas específicas para la evaluación y diagnóstico del TCS así como el establecimiento de unos límites claros y concretos con otros trastornos tales como el TEL o el TEA son una realidad que se recoge en muchos de los trabajos presentados.

Cabe preguntarse cómo se diferencian el TEL, el TEA y el trastorno de la comunicación social y sobre la existencia de unos límites claros entre estas tres alteraciones.

Un estudio reciente (Gibson, Adams, Lockton y Green, 2013) establece que el trastorno pragmático del lenguaje (denominación dada al actual TCS) es un trastorno diferente del autismo por la ausencia de intereses y comportamientos repetitivos y por la presencia de dificultades lingüístico-expresivas. A menudo, los niños con autismo sufren alteraciones de tipo pragmático, pero también se detectaron casos de menores no autistas que presentaban dificultades con la pragmática y la comunicación social. Se le 
prestó atención a dichas problemáticas porque llevaban implícitas alteraciones significativas en la calidad de vida del sujeto, esto es, repercusiones en las relaciones con los pares. En esta investigación se le concede la mayor importancia a las dificultades en la interacción social con pares. Estos problemas son más severos en el TCS y disminuye su presencia y grado de interferencia en la vida cotidiana del individuo tanto en el TEL como en el autismo. Por tanto, el TCS sería un síndrome en sí mismo.

La presencia de comportamientos estereotipados y repetitivos marca la diferencia entre el TCS y el TEL con respeto al espectro autista. En los primeros no se observan los mencionados comportamientos 0 se manifiestan minoritariamente, mientras en el espectro autista son mayoritarios.

La línea divisoria entre autismo, el TEL y el TCS parece no estar muy clara a nivel práctico, más allá de los planteamientos hechos a nivel teórico (Brook y Bowler, 1992). Adams, Lloyd, Aldred \&Baxendale, (2012) concluyen en sus investigaciones que los individuos que sufren TCS presentan déficits en las estructuras lingüísticas menos definidos y más uniformes que en el caso de aquellos con TEL, donde estas dificultades son más variadas y marcadas. Catalogan el TCS como una condición intermedia entre el espectro autista y el trastorno específico del lenguaje, tal como proponen Bishop (2000) y Bishop y Norbury (2002).

Las investigaciones recientes centradas en los aspectos pragmáticos parecen orientarse hacia la delimitación del trastorno de comunicación social (pragmática) y la consideración como un trastorno en sí mismo (síndrome), que presenta unas manifestaciones sintomatológicas concretas y sobre el cual es preciso intervenir de manera específica.

Actualmente los estudios en el campo sobre la pragmática son escasos, muestra de ello es el hecho de que en el período comprendido entre el año 2000 y la actualidad sólo 28 artículos versan exclusivamente sobre los desórdenes en la comunicación social. La investigación sobre el trastorno etiquetado por el DSM-5 apenas ha comenzado y emerge como el grande olvidado dentro de las problemáticas de la comunicación. Este hecho puede venir explicado por la dificultad de detectar de manera precisa la sintomatología asociada al TCS, puesto que puede confundirse con retrasos en el desarrollo, con TEA, fobia social, TEL,... Además los déficits pragmáticos, cuando no son muy marcados y evidentes, tal y como sucede en algunos sujetos adolescentes e incluso adultos, pueden estar ocultos, o pasar desapercibidos debido a conductas compensatorias.

\section{Referencias}

Adams, C. Lloyd, J., Aldred, C. e Baxendale, J. (2006). Exploring the effects of communication intervention for developmental pragmatic language impairments: a signalgeneration study. International Journal of Language and Communication Disorders, 41(1), 41-65.

American Psychiatric Association. (2013). Diagnostic and statistical manual of mental disorders (5th ed.). Washington, DC: Autor.

Baixauli-Fortea, I., Roselló, B. y Miranda-Casas, A. (2004). Evaluación de las dificultades pragmáticas. Estudio de casos. Revista de Neurología, 38 (Supl 1), 69-79.

Belser, R.C. y Dudhalter, V. (2001). Conversational characteristics of children with fragile $\mathrm{X}$ syndrome: repetitive speech. American Journal of Mental Retardation, 106, 28-38.

Bishop, D.V.M. (2000). Pragmatic Language Impairment: A correlate of SLI, a distinct subgroup or part of the autistic continuum?. En D.V.M. Bishop \& L.B. Leonard (Eds.),
Speech and language impairments in children: Causes, characteristics, intervention and outcome (99-113). Philadelphia: Taylor \& Francis.

Bishop, D.V.M. y Norbury, C.F. (2002). Exploring the bordelands of autistic disorder and specific language impairment: a study using standardised diagnostic instruments. Journal of Child Psychology and Psychiatry and Allied Disciplines, 43(7), 917-929. doi: 10.1111/14697610.00114

Botting, N., y Conti-Ramsden, G. (1999). Pragmatic language impairment without autism. SAGE Publications and The National Autistic Society, 3, 371-96.

Botting, N., y Conti-Ramsden, G. (2003). Autism, primary pragmatic diffi -culties and specifi c language impairment: Can we distinguish them using psycholinguistic markers? Developmental Medicine and Child Neurology, 45, 515524.

Brook, S.L. y Bowler, D.M. (1992). Autism by another name - semantic and pragmatic impairments in children. Journal of Autism and Developmental Disorders, 22(1), 61-81. doi: 10.1007/BF01046403

Gibson, J., Adams, C., Lockton, E. \& Green, J. (2013). Social communication disorder outside autism? A diagnostic classification approach to delineating pragmatic language impairment, high functioning autism and specific language impairmet. Journal of Child Psychology and Psychiatry, 54(11), 1186-1197. doi: 10.1111/jcpp.12079

Monfort, I. (2009). Comunicación y lenguaje: bidireccionalidad en la intervención en niños con trastorno de espectro autista. Revista de Neurología, 48(S2), 53-56.

Roqueta, C. y Clemente, R. A. (2010). Dificultades pragmáticas en el trastorno específico del lenguaje. El papel de las tareas mentalistas. Psicothema, 22 (4), 677683.

Sudhalter, V., Morianon, M., Brooks, P. (1992). Expressive semantic deficit in the productive language of males with fragile-X syndrome. American Journal of Medicine Genetics, 43, 65-71.

Taboada, E.M., Rivas, R.M. y López, S. (2012). Desarrollo del lenguaje: dificultades y problemas. En A. Dosil (coord.), Desarrollo cognitivo, afectivo, lingüístico y social (pp. 81-115). Madrid: UDIMA-CEF.

Tannock, M. y Martiussen, R. (2001). Reconceptualizing ADHD. Educational Leadership, 59, 20-25. 